\title{
Computação Desplugada: Um Mapeamento Sistemático da Literatura Nacional
}

\author{
Wilk Oliveira dos Santos ${ }^{1}$, Fernando Carvalho Silva ${ }^{1}$, Lucas Tadeu Hinterholz ${ }^{2}$, \\ Seiji Isotani $^{1}$, Ig Ibert Bittencourt ${ }^{3}$
}

\author{
${ }^{1}$ Laboratório de Computação Aplicada à Educação e Tecnologia Social Avançada (CAEd) \\ Instituto de Ciências Matemáticas e de Computação (ICMC) \\ Universidade de São Paulo (USP) - São Carlos, SP - Brasil. \\ ${ }^{2}$ Curso de Licenciatura em Computação (LC) \\ Departamento de Computação (DC) \\ Universidade de Santa Cruz do Sul (UNISC) - Santa Cruz do Sul - RS - Brasil. \\ ${ }^{3}$ Núcleo de Excelência em Tecnologias Sociais (NEES) \\ Instituto de Computação (IC) \\ Universidade Federal de Alagoas (UFAL) - Maceió, AL - Brasil. \\ \{wilk.oliveira, fernandohc\}@usp.br, sisotani@icmc.usp.br, ig.ibert@ic.ufal.br \\ lhinterholz@gmail.com
}

\begin{abstract}
Resumo. Diante da eminente necessidade de ensinar Ciência da Computação no ensino básico e das dificuldades enfrentadas no seu processo de ensino e aprendizagem, nos últimos anos uma série de estudos têm usado uma estratégia conhecida por Computação Desplugada. No entanto, diversos questionamentos têm sido feitos, por exemplo: i) em quais níveis de ensino a Computação Desplugada tem sido trabalhada?; ii) quais atividades de Computação Desplugada têm sido implementadas?; e iii) como as pesquisas em Computação Desplugada têm sido avaliadas? No intuito de responder essas questões, esse artigo apresenta os resultados de um Mapeamento Sistemático da Literatura sobre Computação Desplugada no Brasil. Os principais resultados indicam que a maioria das abordagens baseiam-se nas atividades do livro "Computer Science Unplugged" e se concentram entre os níveis de ensino fundamental e médio, bem como, é notável a falta de estudos estatísticos sobre a Computação Desplugada no âmbito nacional.
\end{abstract}

\begin{abstract}
Faced with the imminent need to teach Computer Science in basic education and the difficulties faced in its teaching and learning process, in the last few years a series of studies has used a strategy known as Unpluged Computer Science. However, several questions have been asked, for example: i) at which education levels has Unpluged Computer Science been worked? ii) which Unpluged Computer Science activities have been implemented ?; and iii) how has the research on Unpluged Computer Science been evaluated? In order to answer these questions, this article presents the results of a Systematic Mapping of Literature on Unpluged Computer Science on Brazil. The main results indicate that most of the approaches are based on the activities in the "Computer
\end{abstract}


Science Unplugged" book and are concentrated between the primary and secondary school levels, futhermore, the result's lack of statistical studies in the field of Unpluged Computer Science on Brazil.

\section{Introdução}

Desde 2014 com a criação do novo Plano Nacional de Educação (PNE), quando o Ministério da Educação (MEC) definiu vinte metas as serem atingidas pela educação nacional através do PNE, o ensino no Brasil teve seus currículos repensados para os desafios imputados pelo mundo globalizado [BRASIL 2014, Hinterholz and dos Santos 2017, Souza Corrêa and Coelho 2018]. Diante disso, cabe portanto, fazer uma reflexão do que já foi adequado passados quatro anos do lançamento do documento e os desafios do que ainda precisam ser atingidos [Souza Corrêa and Coelho 2018], principalmente no que tange as competências e habilidades inerentes à resolução de problemas [Hinterholz and dos Santos 2017].

Segundo dados do Fundo Nacional de Desenvolvimento da Educação (FNDE) sobre a Prova Brasil (PB) aplicada em estudantes dos anos finais (quinto e nono ano de ensino fundamental e terceiro ano do ensino médio) [Aur and Neto 2018], quase metade destes não apresentam o nível desejável de conhecimento para a sua faixa etária na resolução de problemas. Estes dados trazem um sinal de alerta para os educadores e escolas que recebem estes estudantes no Ensino Básico sem a capacidade de análise e abstração de informações.

Nesse sentido, as situações-problema do contexto atual diferem muito de épocas onde a informação era menos latente, pois, principalmente no mundo do trabalho, busca-se criatividade, dinamismo e agilidade [Silva et al. 2015, Santos et al. 2017a, Santos et al. 2017b]. Os estudantes e futuros profissionais precisam ser estimulados e treinados sob nova ótica que baseia-se em alta capacidade cognitiva, admitindose, assim, a necessidade da competência conhecida como Pensamento Computacional [Bell et al. 1998], considerada uma competência diretamente co-relacionada com a aptidão de decompor um problema dito complexo em pequenas partes, fruto de um refinado pensamento lógico e abstrato [Blikstein 2008].

Nesse contexto, a Computação Desplugada surgiu como uma alternativa viável para o ensino de Ciência da Computação nos mais diferentes níveis de ensino, por se tratar de um conjunto de atividades de computação por meios não digitais, por vezes, somente utilizando material de consumo em uma sala de aula convencional [Bell et al. 1998]. Embora estas práticas relativas ao desenvolvimento do Pensamento Computacional não careçam de suporte das tecnologias, ainda é difícil afirmar como ela está sendo trabalhada nas escolas.

Apesar de saber-se da importância da Computação Desplugada, uma série de questionamentos podem ser feitos a cerca de como os estudos sobre Computação Desplugada têm sido desenvolvidos, aplicados e avaliados no cenário nacional. A fim de entenderse como a Computação Desplugada tem contribuído ou pode contribuir para o ensino de Ciência da Computação, esse artigo apresenta um Mapeamento Sistemático da Literatura (MSL) a cerca das pesquisas nacionais em Computação Desplugada. Os principais resultados desse estudo secundário indicam a falta de estudos no ensino infantil e no ensino médio, bem como, a falta de estudos com abordagem quantitativa e experimental. 


\section{Mapeamento Sistemático da Literatura}

Um MSL é uma forma de identificar, avaliar e interpretar os resultados disponibilizados em uma pesquisa, relacionados a uma questão de pesquisa, tópico, área ou fenômeno. $\mathrm{O}$ principal objetivo de conduzir um MSL consiste em recolher elementos de uma pesquisa [Kitchenham 2004]. Essa seção tem por objetivo apresentar o protocolo desse MSL.

\subsection{Protocolo do Mapeamento}

Para a realização desse MSL, foram utilizadas as diretrizes propostas por [Kitchenham 2004]. O processo inclui atividades que podem ser agrupadas em três fases: Planejamento, onde são definidas as Questões de Pesquisa (QP), a string de busca, os Critérios de Inclusão (CI) e Exclusão (CE), e as bases de pesquisa; Condução, onde a string de busca é utilizada nas bases de pesquisa selecionadas, e são aplicados os CI e CE; e Relatório dos Resultados, onde é realizado uma exposição dos resultados encontrados. Todo o protocolo desse estudo foi definido e executado por meio da participação de três profissionais com expertise em Ensino de Ciência da Computação, Computação Desplugada e na condução de estudos secundários.

\subsubsection{Objetivos}

Esse MSL tem como principal objetivo compreender o cenário de pesquisa em Computação Desplugada no Brasil. Para isso, uma série de QP foram levantadas.

\subsubsection{Questões de Pesquisa}

Com base no objetivo anteriormente descritos, as seguintes QP foram levantadas:

1. QP1: Em quais níveis de ensino a Computação Desplugada tem sido trabalhada (ensino fundamental, ensino médio, ensino superior)?

2. QP2: Quais atividades de Computação Desplugada têm sido implementadas (adaptadas, desenvolvidas sob medida, mistas)?

3. QP3: Como as pesquisas em Computação Desplugada têm sido avaliadas (quantitativamente, qualitativamente, mista)?

\subsubsection{String de Busca}

A string de busca tem como objetivo principal reunir os termos que conjuguem o tópico a ser abordado [Kitchenham 2004]. A string de busca desse estudo objetivou reunir os principais termos associados a Computação Desplugada. Por se tratar de uma pesquisa que visa analisar um determinado nicho de pesquisa no cenário de pesquisa nacional, bem como, pelo fato das revistas e conferências nacionais permitirem artigos escritos nos idiomas Português, Inglês e Espanhol, as palavras-chave da string de busca, foram replicadas nesses três idiomas: ("computação desplugada” OR "informática desplugada”OR "programação desplugada” OR "atividades desplugadas” OR “computer science unplugged”OR "unplugged class" OR "informática desenchufada”). 


\subsubsection{Fontes de Pesquisa}

A estratégia de busca utilizada incluiu apenas bases de dados eletrônicos como fontes de pesquisa para o desenvolvimento deste trabalho. De acordo com a recomendação dos especialistas, as seguintes bases de dados eletrônicas foram pesquisadas: Revista Novas Tecnologias na Educação (RENOTE) ${ }^{1}$, Revista Brasileira de Informática na Educação (RBIE) ${ }^{2}$, Simpósio Brasileiro de Informática na Educação (SBIE) 3 , Workshop de Informática na Escola (WIE) $)_{4}^{4}$. Workshops do Congresso Brasileiro de Informática na Educação (WCBIE) ${ }^{5}$, Workshop sobre Educação em Computação (WEI) ${ }^{6}$. Essas são principais bases nacionais que agregam trabalhos da comunidade Brasileira de Informática na Educação e Educação em Computação. Apenas o WEI não permite busca automática (por meio da string de busca), assim, no WEI foi conduzida uma busca manual extensiva por meio da leitura de titulo e resumo de todos os artigos publicados nos anais do evento (separadamente pelos três pesquisadores).

\subsubsection{Critérios de Inclusão e Exclusão}

O objetivo de definir os critérios de inclusão e exclusão é identificar aqueles estudos primários que fornecem evidência direta sobre as QP previamente definidas, bem como para reduzir a probabilidade de algum viés [Kitchenham 2004]. Foram selecionados para inclusão no MSL estudos primários (consideramos como estudos primários aqueles que apresentam algum tipo de estudo sobre Computação Desplugada no contexto nacional), que de alguma forma apresentaram contribuições no contexto da Computação Desplugada. Em função de sabidamente área de Computação Desplugada ter surgido em meados de 2012, optou-se por buscar-se estudos publicados entre Janeiro de 2012 e Junho de 2018. Os estudos excluídos foram os estudos secundários e terciários, artigos resumidos, sem revisão dupla, duplicados, artigos redundantes da mesma autoria, artigos de posicionamento e que seu foco não estava apoiado na pesquisa sobre Computação Desplugada. Um resumo dos critérios de inclusão e exclusão é apresentado na Tabela 1.

Tabela 1. Crítérios de Inclusão e Exclusão

\begin{tabular}{c|c}
\hline Critérios de Inclusão & Critérios de Exclusão \\
\hline $\begin{array}{c}\text { Estudos Estudos primários } \\
\text { relacionados ao domínio de pesquisa }\end{array}$ & Estudos com menos de 5 páginas \\
\hline Estudos publicados entre 2012 e 2018 & Estudos duplicados e/ou Estudos redundantes \\
\hline
\end{tabular}

\subsubsection{Extração de Dados}

Seguindo o protocolo de [Kitchenham 2004], a extração dos dados foi feita com base na leitura individual de cada artigo selecionado. Na primeira etapa, foram lidos os títulos

\footnotetext{
1 http://seer.ufrgs.br/renote

2 http://www.br-ie.org/pub/index.php/rbie

3 http://www.br-ie.org/pub/index.php/sbie

4 http://www.br-ie.org/pub/index.php/wie

5 http://www.br-ie.org/pub/index.php/wcbie

6http://www.sbc.org.br/eventos/csbc
} 
e resumos de todos os artigos encontrados por meio da pesquisa automática nas bases investigadas. Essa etapa teve por objetivo selecionar apenas os trabalhos que de fato se relacionassem ao tópico de Computação Desplugada. Na segunda etapa, foram lidos os textos completos de todos os artigos selecionados nas etapas anteriores, a fim de extrair as informações necessárias para compor MSL. As informações extraídas dos artigos foram: i) título; ii) resumo; iii) palavras-chave; $i v$ ) autores; $v$ ) instituições; vi) abordagem proposta; vii) domínio/disciplina; viii) nível; $i x$ ) atividade; e $x$ ) metodologia de avaliação.

\section{Resultados}

No intuito de apresentar os resultados deste MSL, organizamos cada resultado em subseções especificas apresentadas a seguir de acordo com as QP previamente definidas. Inicialmente, a Figura 1 mostra o processo de filtragem dos artigos, inciando com a busca em cada uma das bases escolhidas e culminando com a seleção dos estudos finais incluídos neste mapeamento (15 estudos). A Tabela 2 apresenta uma lista com todos os estudos selecionados e a Figura 2 mostra a tendência de publicação nos últimos anos com perspectiva de crescimento para os próximos anos. Uma sumarização dos resultados contendo todo processo de filtragem dos artigos pode ser acessado clicando aqui. Além disso, cada um dos artigos originais selecionados pode ser acessado de maneira completa e automática clicando em seu código de identificação $(i d)$, disponível na primeira coluna da Tabela 2 ou em qualquer outro local que o artigo seja referenciado ao longo do texto.

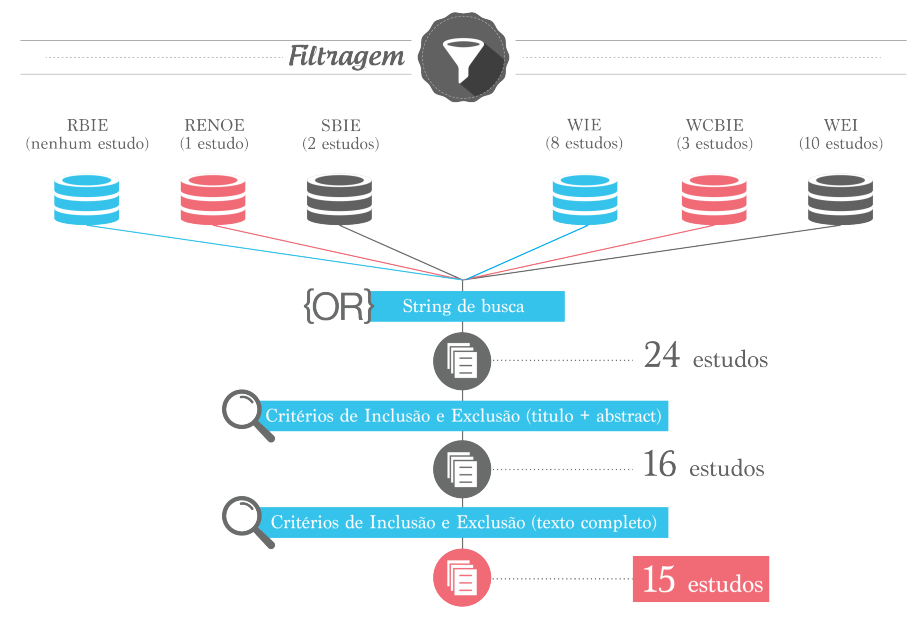

Figura 1. Filtragem dos estudos

Tabela 2: Lista de Artigos

\begin{tabular}{c|l}
\hline \multicolumn{1}{l|}{$\mathbf{I d}$} & Titulo \\
\hline $\mathrm{E} 1$ & $\begin{array}{l}\text { Um Relato de Experiências de Estagiários da Licenciatura em Computação } \\
\text { com o Ensino de Computação para Crianças }\end{array}$ \\
\hline$\overline{\mathrm{E} 2}$ & Bem Mais que os Bits da Computação Desplugada \\
\hline \hline $\mathrm{E}$ & $\begin{array}{l}\text { Oficinas itinerantes de scratch e computação desplugada para professores } \\
\text { como apoio ao ensino de computação - um relato de experiência }\end{array}$ \\
\hline \hline E4 & $\begin{array}{l}\text { Desenvolvimento do Pensamento Computacional: Um relato de atividade junto ao } \\
\text { Ensino Médio, através do Estágio Supervisionado em Computação III }\end{array}$ \\
\hline
\end{tabular}




\begin{tabular}{|c|c|}
\hline E5 & Proposta de atividade para o quinto ano do ensino fundamental: Algoritmos Desplugados \\
\hline E6 & $\begin{array}{l}\text { Experiência Prática Interdisciplinar do Raciocínio Computacional } \\
\text { em Atividades de Computação Desplugada na Educação Básica }\end{array}$ \\
\hline E7 & $\begin{array}{l}\text { Representação e Análise de Dados no Quinto Ano do Ensino Fundamental: } \\
\text { Proposta de Atividade e Relato de Aplicação }\end{array}$ \\
\hline E8 & $\begin{array}{l}\text { Pensamento Computacional: Uma Proposta de Ensino com Estratégias } \\
\text { Diversificadas para Crianças do Ensino Fundamental }\end{array}$ \\
\hline E9 & $\begin{array}{l}\text { A Contribuição do Projeto Berçário de Hackers na Alfabetização Matemática de } \\
\text { Crianças em Fase Pré-Operatória }\end{array}$ \\
\hline E10 & $\begin{array}{l}\text { Proposta e Adaptação de Atividades Desplugadas para o Ensino de Computação } \\
\text { na Educação Básica }\end{array}$ \\
\hline E11 & $\begin{array}{l}\text { Experiencias de Ensino da Computação Desplugada em Diferentes Series da } \\
\text { Educação Fundamental Maior }\end{array}$ \\
\hline$\overline{\mathrm{E} 12}$ & Um Relato de Experiência do Uso da Técnica Computação Desplugada \\
\hline E13 & $\begin{array}{l}\text { O ensino de conceitos computacionais para alunos do ensino médio: relato de } \\
\text { experiência de uma gincana e das estratégias utilizadas pelos alunos na } \\
\text { resolução das atividades desplugadas }\end{array}$ \\
\hline E14 & $\begin{array}{l}\text { O Ensino de Computação na Educação Básica apoiado por Problemas: } \\
\text { Práticas de Licenciandos em Computação }\end{array}$ \\
\hline$\overline{E 15}$ & Computação Desplugada no Ensino de Bancos de Dados na Educação Superior \\
\hline
\end{tabular}

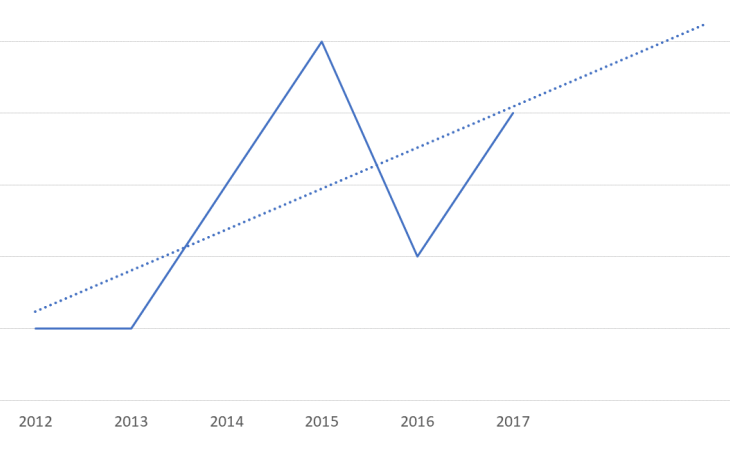

Figura 2. Evolução das Publicações

\subsection{QP1: Em quais níveis de ensino a Computação Desplugada tem sido estudada?}

No intuito de responder essa QP, extraímos de cada artigo selecionado o nível de ensino (e.g., ensino fundamental ou ensino médio) no qual o estudo foi conduzido, por exemplo. Foi possível identificar o nível de ensino em todos os artigos selecionados. Alguns estudos conduziram sua pesquisa em mais de um um nível de ensino, existindo nos estudos selecionados as chamadas abordagens mistas, onde uma mesma abordagem é aplicada em mais de um nível de ensino. A Tabela 3 sumariza os resultados obtidos apresentando ainda o nível em cada um dos estudos.

É possível perceber que a maioria dos estudos (nove estudos) foram conduzidos no contexto do ensino fundamental (considerando que alguns estudos conduziram abor- 
Tabela 3. Níveis de Ensino

\begin{tabular}{|c|c|}
\hline Nível & Estudos \\
\hline Educação infantil & $\overline{\mathrm{E} 9}$ \\
\hline Ensino fundamental & E1;E2;E5; E7; E8; E14 \\
\hline Ensino fundamental e médio & E6; E11; E12 \\
\hline Ensino médio & E4; E13 \\
\hline Ensino superior & E3; E10; E15 \\
\hline
\end{tabular}

dagens mistas). Apesar de os autores não disponibilizarem essas informações, esse resultado possivelmente se baseia no material Computational Thinking in K-12 Education leadership toolkit desenvolvido em conjunto com a Computer Science Teachers Association (CSTA), National Science Foundation (NSF) e International Society for Technology in Education (ISTE) [Stephenson et al. 2012], que propõem que ao final do ensino médio os alunos devem demonstrar habilidades básicas para resolução de problemas gerais baseado no fundamentos e técnicas da Ciência da Computação, também conhecidas como Pensamento Computacional [Wing 2006]. Ressalta-se que apenas um estudo foi conduzido no ensino infantil, deixando uma lacuna no uso de Computação Desplugada justamente em uma época em que as crianças começam a desenvolver o raciocínio lógico acerca de determinadas situações cotidianas [Rappaport 1981].

\subsection{QP2: Quais atividades de Computação Desplugada têm sido conduzidas?}

Para responder essa QP, extraiu-se de cada artigo exatamente qual atividade foi conduzida ou proposta no estudo. Em apenas dois estudos não foi possível identificar quais atividades foram conduzidas durante a realização do estudo. Alguns estudos conduziram mais de uma atividade dentro da mesma abordagem. A Tabela 4 apresenta as atividades que foram conduzidas nos estudos selecionados.

Tabela 4. Atividades conduzidas

\begin{tabular}{l|l|}
\hline \multicolumn{2}{c}{ Tabela 4. Atividades conduzidas } \\
\hline Atividade & Estudo \\
\hline Atividade autoral & E4; E8; E15 \\
\hline $\begin{array}{l}\text { Atividade proposta } \\
\text { por Andrate et al. (2013) }\end{array}$ & E7 \\
\hline $\begin{array}{l}\text { Atividades do livro } \\
\text { "Computer Science Unplugged" }\end{array}$ & E1; E2; E3; 6 6) E10; E11; E12; E13; E14 \\
\hline Não identificado & E5; E9 \\
\hline
\end{tabular}

A resposta à essa QP nos permitiu identificar que a maioria dos estudos teve como objetivo popularizar o ensino de Computação nas escolas e desenvolver o pensamento computacional nos alunos. Dentre os estudos selecionados, nove utilizaram-se de atividades presentes no livro Computer Science Unplugged, como "A mágica de virar cartas", para detecção e correção de erros e "Contando os pontos" para o ensino de números binários. A maioria destas atividades são de caráter introdutório e podem ser encontradas no capítulo I do livro que aborda representação da informação.

Além disso, três outros estudos propuseram atividades autorais desenvolvida por grupos de alunos e pesquisadores, onde atividades lúdicas foram utilizadas como ferramentas para o ensino de conceitos introdutórios, como o uso de origamis ou quebra 
cabeças para ensinar conceitos de sequenciamento de processos em algoritmos. Um único estudo aplicou ainda uma atividade proposta por [Andrade et al. 2013] com o objetivo de estimular a coleta e organização de dados por parte dos alunos, e dois estudos entre os trabalhos selecionados não identificaram o tipo de atividades realizadas.

\subsection{QP3: Como as pesquisas em Computação Desplugada tem sido avaliada?}

Para responder essa QP, extraímos de cada um dos estudos selecionados, como a atividade proposta ou aplicada foi avaliada pelos pesquisadores. Nesse caso, em apenas um estudo não foi possível identificar como foi conduzida a avaliação. A Tabela 5 apresenta qual o tipo de avaliação conduzida em cada estudo.

Tabela 5. Avaliação das pesquisas

\begin{tabular}{|c|c|}
\hline Tipo de pesquisa & Estudos \\
\hline Pesquisa quali-quanti & E13; E15 \\
\hline Pesquisa qualitativa & 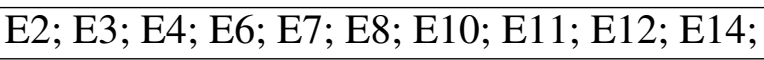 \\
\hline Pesquisa quantitativa & $\overline{\mathrm{E} 1} ; \overline{\mathrm{E} 5}$ \\
\hline Não identificado & $\overline{\mathrm{E} 9}$ \\
\hline
\end{tabular}

A resposta a essa QP nos mostra que a grande maioria dos estudos conduzidos nesse domínio possuem apenas avaliação qualitativa, em geral, partindo da observação dos próprios autores dos artigos ou de professores externos com relação a atividade aplicada (estudos E4 e E14), ou ainda da aplicação de questionários para avaliar a aceitação dos alunos em relação as atividades (estudos E2, E7, E8 e E12). Além disso, entre os trabalhos selecionados quatro estudos conduziram uma avaliação quantitativa dos resultados analisando a aprendizagem dos alunos em testes de conhecimento do tipo pós-teste (estudos E1, E5, E13 e E15).

No entanto esses trabalhos não citam o uso de pré-testes ou testes de retenção, não permitindo uma análise mais completa dos resultados. Além disso, entre os estudos selecionados apenas dois estudos apresentaram abordagens qualitativa e quantitativa (E13 e E15), onde são analisados não apenas as opiniões dos participantes sobre a experiência vivenciada, mas também o impacto da abordagem na aprendizagem dos alunos. Esses resultados deixam claro a necessidade de estudos estatísticos neste domínio de pesquisa e a necessidade da condução deste tipo de estudo.

\subsection{Discussões Gerais}

Os resultados desse MSL indicaram que a maioria dos estudos foram conduzidos no ensino fundamental e médio, em detrimento a abordagens no ensino fundamental. Sabese no entanto, que é justamente nessa idade em que os estudantes estão cursando o ensino básico, que o raciocínio logico das crianças começam a se desenvolver [Wing 2006] podendo ser intrinsecamente potencializado por atividades especificas ligadas ao desenvolvimento do Pensamento Computacional [Blikstein 2008]. Nesse sentido, é possível perceber a necessidade de conduzir-se estudos aplicando a Computação Desplugada em diferentes níveis de ensino, especialmente no ensino infantil, a fim de potencializar o desenvolvimento cognitivo e do pensamento computacional nas crianças, ainda no primeiro ciclo de aprendizagem formal. 
Ao mesmo tempo, é possível perceber que a maioria das atividades são baseadas em um único livro, em detrimento a propostas de novas atividades de Computação Desplugada. Apesar da importância das atividades propostas no livro, destaca-se a importância de conduzir-se novos estudos que proponham e avaliem novas atividades e abordagens para o ensino de Ciência da Computação por meio de atividades da Computação Desplugada, de modo promover-se o Pensamento Computacional nos estudantes de ensino básico.

Finalmente, os resultados mostram ainda que a maioria dos estudos passaram apenas por avaliações qualitativas, em geral, partindo da observação direta de algum professor-aluno que acompanhou a aplicação das atividades. Assim, destaca-se ainda importância da condução de estudos estatísticos que possam contribuir com resultados quantitativos/experimentais sobre os efeitos da Computação Desplugada no ensino de Ciência da Computação. Esse tipo de estudo poderá trazer para a comunidade uma nova fase, com resultados validades e passiveis de replicação e acompanhamento em outros cenários.

\section{Ameças a Validade}

Para organizar essa seção, as ameaças à validade foram classificadas usando as categorias: Interna, Externa, Construção e Conclusão [Wohlin et al. 2012]. De modo a mitigar ameaças a Validação Interna desse trabalho, onde possíveis decisões subjetivas podem ter ocorrido durante a escolha dos artigos e a extração de dados, o processo foi realizado através do mecanismo peer-to-peer, onde cada artigo foi analisado por dois pesquisadores e casos de decisões contraditórias foram definidos por meio de um terceiro pesquisador. Com relação a Validação Externa o processo de pesquisa foi definido após várias pesquisas de teste validado com o consenso de todos os pesquisadores além disso.

Os termos utilizados nesse trabalho foram escolhidos com base nas sugestões de pesquisadores (pesquisadores com experiência nesta área) e de acordo com as pesquisas recentes sobre Computação Desplugada, mitigando assim, possíveis ameaças a Validação de Construção. Por fim, é possível que alguns estudos excluídos nesse MSL devessem ser incluídos, sendo esta uma possível Validação da Conclusão deste trabalho. No entanto, para mitigar essa ameaça, o processo de seleção e os critérios de inclusão e exclusão foram cuidadosamente elaborados, discutidos e revisados pelos pesquisadores, a fim de minimizar o risco de exclusão de estudos relevantes.

\section{Considerações Finais}

Esse artigo apresentou um MSL sobre Computação Desplugada no cenário nacional. Os resultados indicaram a que a maioria dos estudos têm sido conduzidos no contexto do ensino fundamental, com a mesma escolha de material didático, Computer Science Unplugged, e com avaliações qualitativas dos resultados obtidos. As principais contribuições desse trabalho estão direcionadas ao ensino de Ciência da Computação. Espera-se e recomenda-se como trabalhos futuros a condução de estudos que possam ir de encontro aos desafios identificados por meio dos resultados desse estudo e destacados em nossas discussões. 


\section{Referências}

[Andrade et al. 2013] Andrade, D., Carvalho, T., Silveira, J., Cavalheiro, S., Foss, L., Fleischmann, A. M., Aguiar, M., and Reiser, R. (2013). Proposta de atividades para o desenvolvimento do pensamento computacional no ensino fundamental. In Anais do Workshop de Informática na Escola, volume 1, page 169.

[Aur and Neto 2018] Aur, B. A. and Neto, J. J. S. (2018). Base nacional comum curricular. Boletim Técnico do Senac, 44(1).

[Bell et al. 1998] Bell, T. C., Witten, I. H., and Fellows, M. (1998). Computer Science Unplugged: Off-line activities and games for all ages. Citeseer.

[Blikstein 2008] Blikstein, P. (2008). O pensamento computacional e a reinvenção do computador na educação. Education \& Courses.

[BRASIL 2014] BRASIL, C. (2014). Planejando a próxima década: conhecendo as 20 metas do plano nacional de educação. Brasília: Ministério da Educação.

[Hinterholz and dos Santos 2017] Hinterholz, L. and dos Santos, W. O. (2017). Aprendizagem baseada em projetos: Relato de introdução da lógica no ensino fundamental. In Anais do Workshop de Informática na Escola, volume 23, page 1154.

[Kitchenham 2004] Kitchenham, B. (2004). Procedures for performing systematic reviews. Keele, UK, Keele University, 33(2004):1-26.

[Rappaport 1981] Rappaport, C. R. (1981). Psicologia do desenvolvimento: teorias do desenvolvimento conceitos fundamentais. EPU.

[Santos et al. 2017a] Santos, W. O., Hinterholz, L., and Silva, C. (2017a). Licenciatura em computação: Desafios e oportunidades na perspectiva do professor. In Anais do Workshop de Informática na Escola, volume 23, page 705.

[Santos et al. 2017b] Santos, W. O., Silva, C., and Hinterholz, L. (2017b). Licenciatura em computação: Desafios e oportunidades na perspectiva do estudante. In Anais do Workshop de Informática na Escola, volume 23, page 885.

[Silva et al. 2015] Silva, S. d., Ferreira, A., Souza, A., Galdino, E., Oliveira, M., Neto, S., and Oliveira, W. (2015). Relato de experiência de ensino de computação no ensino fundamental em estágio supervisionado da universidade de pernambuco no campus garanhuns. In $23^{\circ}$ Workshop sobre Educação em Computação, pages 1-10.

[Souza Corrêa and Coelho 2018] Souza Corrêa, S. and Coelho, A. L. (2018). O atual plano nacional de educação: uma análise das metas e estratégias para a educação básica. DOXA: Revista Brasileira de Psicologia e Educação, 20(1).

[Stephenson et al. 2012] Stephenson, C., Cooper, S., Owens, B., and Gal-Ezer, J. (2012). The new csta $\mathrm{k}-12$ computer science standards.

[Wing 2006] Wing, J. M. (2006). Computational thinking. Commun. ACM, 49(3):33-35.

[Wohlin et al. 2012] Wohlin, C., Runeson, P., Höst, M., Ohlsson, M. C., Regnell, B., and Wesslén, A. (2012). Experimentation in software engineering. Springer Science \& Business Media. 